\title{
Automated Human Habenula Segmentation from T1-weighted Magnetic Resonance Images using V- Net
}

Joo-won $\mathrm{Kim}^{1,2,{ }^{*}}$, Junqian $\mathrm{Xu}^{1,2}$

${ }^{1}$ Department of Radiology, Baylor College of Medicine, Houston, Texas 77030

${ }^{2}$ Department of Psychiatry, Baylor College of Medicine, Houston, Texas 77030

* Correspondence: joo-won.kim@bcm.edu

\begin{abstract}
The Habenula $(\mathrm{Hb})$, a small bilateral midbrain structure, plays an important role in aversion and reward processing. Despite increasing interest in imaging human $\mathrm{Hb}$ structure and function, it is challenging to define the $\mathrm{Hb}$ in magnetic resonance imaging (MRI) due to its small size and low anatomical contrast to the surrounding thalamus. In this study, we developed a deep learning-based human $\mathrm{Hb}$ segmentation model. This model was trained and tested using about a thousand subjects' 3 T T1-weighted images with $\mathrm{Hb}$ labels from our previous myelin content-based $\mathrm{Hb}$ segmentation. The predicted $\mathrm{Hb}$ segmentation showed high similarity and small surface distance to the label; the average (across test subjects) dice similarity coefficient, mean distance, and Hausdorff distance were $0.79,0.22 \mathrm{~mm}$, and $1.63 \mathrm{~mm}$, respectively. We also demonstrated out-of-sample robustness using other 7T and 3T MRI datasets. The proposed $\mathrm{Hb}$ segmentation method is automated, objective, fast, robust, and reliable, relieving the requirement of $\mathrm{T} 2$-weighted images in the established myelin content-based $\mathrm{Hb}$ segmentation to suite a broader range of MRI studies.
\end{abstract}




\section{Introduction}

The Habenula $(\mathrm{Hb})$ is a small bilateral midbrain structure located next to the dorsomedial thalamus and the third ventricle (Namboodiri et al., 2016). The $\mathrm{Hb}$ consists of two subregions, medial and lateral $\mathrm{Hb}$, which are functionally and anatomically distinct (Benarroch, 2015; Díaz et al., 2011). The Hb plays a crucial role in aversion and reward processing, and growing evidence suggests its implication in psychiatric disorders including depression and addiction (Fore et al., 2018; Hikosaka, 2010). This led to an increasing interest in human $\mathrm{Hb}$ structure and function research recently.

However, in vivo human $\mathrm{Hb}$ imaging is challenging because of its small size (about $30 \mathrm{~mm}^{3}$ in each hemisphere) (Ranft et al., 2010) and low anatomical magnetic resonance imaging (MRI) contrast to the surrounding thalamus in any single structural MRI modality, e.g., T1-weighted (T1w) or T2-weighted (T2w) images. However, its contrast to the thalamus can be increased by combining high-resolution 3D $\mathrm{T} 1 \mathrm{w}$ and $\mathrm{T} 2 \mathrm{w}$ images into T1w-over-T2 $\mathrm{w}$ ratio (T1w/T2w) images due to its high myelin content (Glasser et al., 2013). Leveraging this enhanced $\mathrm{Hb}$ contrast in T1w/T2 $\mathrm{w}$ image, we have developed an automated $\mathrm{Hb}$ segmentation method using this increased contrast, saying myelin content-based segmentation (Kim et al., 2018, 2016). This method can be applied to either functional (Ely et al., 2019, 2016) or volumetric(Jha et al., 2021; Schafer et al., 2018) analysis.

The requirement of high-resolution 3D T1w and T2w images of our T1w/T2w segmentation method, nevertheless, limits its wider applicability, because not until recently, the vast majority of neuroimaging research projects only acquired T1w anatomical images. Manual $\mathrm{Hb}$ segmentation (Carceller-Sindreu et al., 2015; Savitz et al., 2011; Schmidt et al., 2016) or geometric Hb segmentation using surrounding landmark structures (Lawson et al., 2013) have been widely employed if only T1w images were available. However, these manual $\mathrm{Hb}$ segmentation methods are laborious and subjective. Recently, other automated $\mathrm{Hb}$ segmentation methods utilizing only T1 $\mathrm{w}$ images have been proposed, such as registration-based multiatlas-driven $\mathrm{Hb}$ segmentation (Germann et al., 2020) and deep learning-based Hb segmentation (Lim et al., 2021). Germann's registration-based method depends on the quality of the registration to their atlases and takes a long process time. Lim's deep learning method was trained and tested using their 69 participants' axial 7T T1 map slices containing $\mathrm{Hb}$, and therefore, it is more likely a project-specific model requiring 7T T1 maps and manual pre-selection of slices.

Deep learning has been widely applied to various image segmentation applications because of its versatility, remarkable performance, and short processing time. There has been much advancement in medical image segmentation using deep learning for small anatomical structures such as spinal cord gray matter 2D segmentation (Perone et al., 2018). U-Net is the most prevalent 2D image segmentation deep-learning model, and it has shown good performance on medical image segmentation (Ronneberger et al., 2015). However, segmenting small tear-shaped structures like $\mathrm{Hb}$ in 3D is much preferred to 2D. Application of 3D deep learning segmentation is still rare in medical imaging, probably due to a lack of reference segmentation for these small structures for training 3D deep learning models.

Deep learning-based segmentation requires, in general, more than thousands of training data to achieve high accuracy and to avoid overfitting because of its complex network structure. It is difficult to create several such labels (i.e., reference segmentation) from medical imaging data since many will require manual work. In order to solve this limitation, image data augmentation is commonly used by slightly modifying image data to increase the amount of data. Such augmentation may include flipping images, adding noises, transforming images, zooming, and so on. Although these methods artificially increase 
the number of labels, one still needs a large number of subject data to address subject-wise nature variability. By leveraging a large amount of readily available reference 3D $\mathrm{Hb}$ segmentations using our T1w/T2w segmentation method (Jha et al., 2021), we are uniquely positioned to use these for 3D deep learning model training.

Here, we introduce an automated, objective, fast, and robust $\mathrm{Hb}$ segmentation on T1w images using 3D deep learning to overcome the limitations of previous methods. We adopted V-Net (Milletari et al., 2016), a 3D version of $\mathrm{U}-\mathrm{Net}$, and trained the model with about a thousand reference $\mathrm{Hb}$ segmentation and evaluated its performance. In addition, we applied the trained model to other datasets to show its robustness.

\section{Methods}

\subsection{Subjects and MRI acquisition}

Training, validation, and test data were obtained from the Human Connectome Project Young Adult (HCP-YA) S1200 (Van Essen et al., 2012). The HCP-YA S1200 participants were healthy subjects ( $n=1113)$ between 22-37 years old. 3T T1w and T2w structural images were acquired at $0.7 \mathrm{~mm}$ isotropic resolution (Glasser et al., 2013).

Other datasets to evaluate the Hb segmentation performance included CamCAN data (Shafto et al., 2014; Taylor et al., 2017), and Mount Sinai 7T data. The CamCAN data are of 652 subjects with a wide age range (18-88 years), and myelin content-based segmentation is applicable to them. They contain T1 $\mathrm{w}$ and T2w images with $1 \mathrm{~mm}$ isotropic resolution from 3T Trio (Siemens) with the following parameters; T1w: 3D MPRAGE, TR/TE/TI = 2250/2.99/900 ms, FA = $9^{\circ} ; \mathrm{T} 2 \mathrm{w}: 3 \mathrm{D}$ SPACE, TR/TE/TI = 2800/408/900 ms. The Mount Sinai 7T data contain MP2RAGE (Marques et al., 2010) UNI images with $0.5 \mathrm{~mm}$ isotropic resolution from 7T Magnetom (Siemens) with the following parameters; TR/TE/TI1/TI2 $=5000 / 5.75 / 900 / 2780 \mathrm{~ms}, \mathrm{FA} 1 / \mathrm{FA} 2=5 / 3^{\circ}$, covering most of the brain.

\subsection{Reference $\mathrm{Hb}$ segmentation}

To use as training, validation, and test labels for the deep learning process, we applied the previously proposed fully-automated version of myelin content-based Hb segmentation (Kim et al., 2018) from ACPC aligned T1w, T2w, and T1w/T2w images. The resulting segmentations were probability maps ranging from 0 to 1 . We segmented $\mathrm{Hb}$ from the HCP-YA S1200 and CamCAN data, and then we visually inspected the segmentation to exclude unsatisfactory segmentation such as underestimation or overestimation (Jha et al., 2021; Kim and Xu, 2018). 




Figure 1. Overview of deep learning model training and prediction. Top: training process including $\mathrm{Hb}$ segmentation (Kim et al. 2016 using T1w, T2w, and T1w/T2w images) and image augmentation (random noise, crop, and $x$-flip). Bottom: prediction process including crop, prediction, and postprocessing.

\subsection{Machine learning setup}

See Fig. 1 for an overview of our machine learning training and prediction process

We randomly separated the HCP-YA S1200 data with satisfactory Hb segmentation into training, validation, and test sets ( $n=717,127$, and 157, respectively). The inputs were AC-PC aligned T1w images of the subjects, and the labels were the corresponding $\mathrm{Hb}$ segmentation after applying empirical threshold 0.3 to the $\mathrm{Hb}$ segmentation probability maps.

To reduce the input image size for memory limitation, we cropped the T1w images to $64 \times 64 \times 64$ voxels centered at random locations around the $\mathrm{Hb}$ (center $\mathrm{x}, \mathrm{y}$, and $\mathrm{z}$ coordinates were within 110-146, 129165 , and $87-123$ ranges, respectively, in the total $260 \times 311 \times 260$ voxel space). To increase the number of training sets, we employed some image data augmentation methods during the training process. First, both with- and without-bias-field-correction (Glasser et al., 2013) T1w images were used which have the 
same shape but different intensities. Second, the center of the crop was random, resulting in the shift of the $\mathrm{Hb}$ location. Third, the images were randomly left-right-flipped. Fourth, random noise was added.

While a typical deep learning-based image classification network monotonically decreases the image dimension (matrix dimension), V-Net or U-net contains steps to recover the matrix dimension (Fig. 2, expansion) after decreasing steps (Fig. 2, contraction). At each recovering step, corresponding decreasing step's matrices are concatenated. Through these recovering steps, the output can have the same dimension as the input.

Our V-Net model was implemented in Keras 2.4.3 (Chollet and others, 2015) with tensorflow-gpu 2.2.0 (Martín Abadi et al., 2015) and CUDA 10.1 (NVIDIA, 2019) backend, modified from a V-Net implementation in GitHub (https://github.com/MiguelMonteiro/VNet-Tensorflow). The detailed parameters were: number of levels $=3$, number of convolutions $=(1,2,3)$, number of channels $=(32$, $64,128)$, number of bottom convolutions $=3$, activation function before the last layer $=$ Parametric Rectified Linear Unit (PReLU), (He et al., 2015), dropout rate $=0.5$, number of epochs $=50$, optimizer $=$ Adam (Kingma and $\mathrm{Ba}, 2017$ ), initial learning rate $=0.001$, loss function $=$ dice similarity coefficient (DSC), (Dice, 1945). We trained the model on a NVIDIA ${ }^{\circledR}$ TeslaTM T4 16GB system.

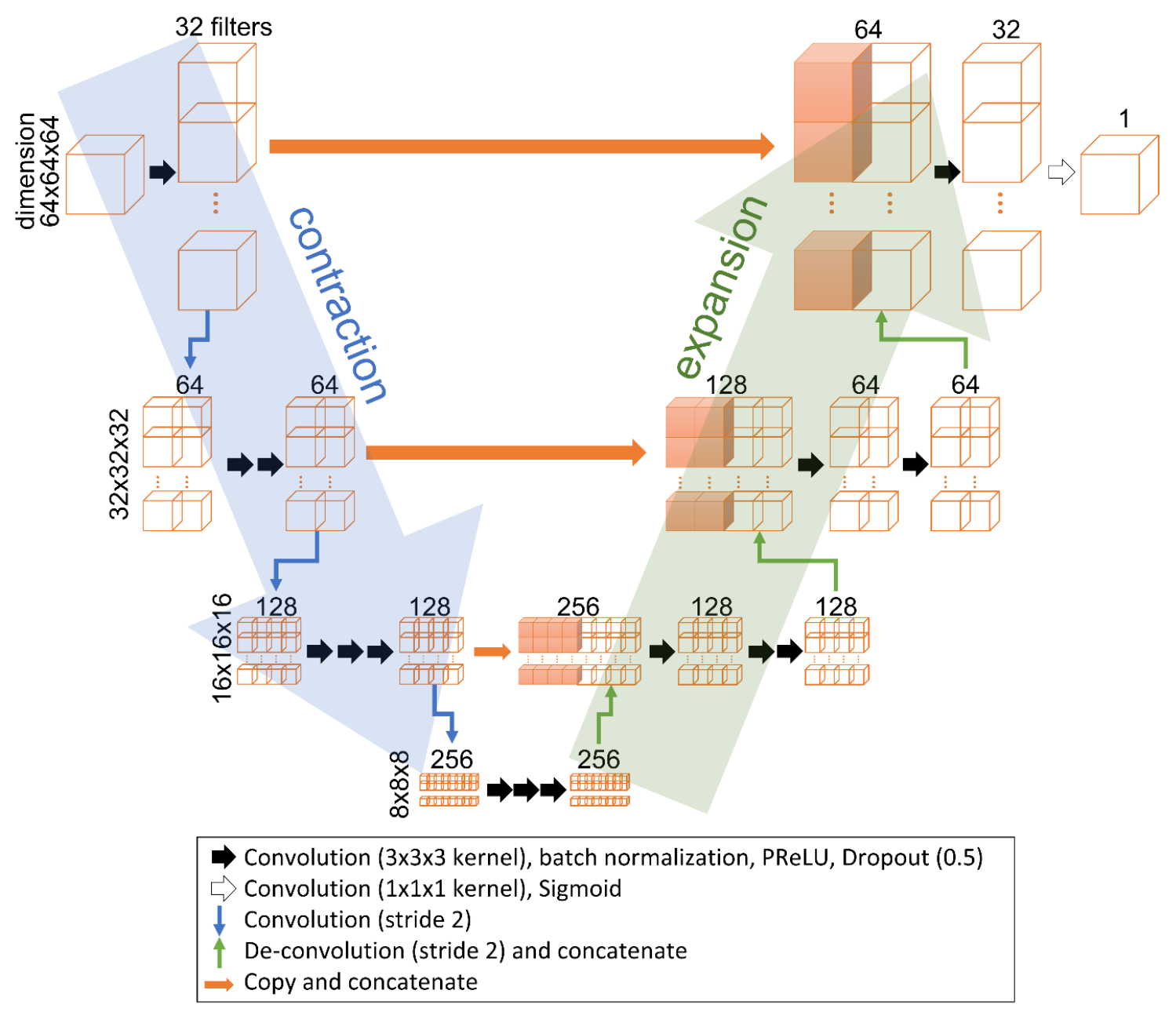


Figure 2. Schematic representation of the model network architecture. The left half is the contraction part reducing each volume dimension from $64 \times 64 \times 64$ down to $8 \times 8 \times 8$ voxels, and the right half is the expansion part enlarging the dimension up to the original volume, $64 \times 64 \times 64$ voxels. The number of filters increases at the contraction part and decreases at the expansion part. The black arrows represent a set of 3-D convolution with $3 \times 3 \times 3$ kernel size preserving the input dimension, batch normalization, PReLU activation function, and dropout operations. The white arrow represents a set of 3-D convolution with $1 \times 1 \times 1$ kernel size and Sigmoid activation function operations. The downward blue arrows represent 3-D convolution with stride 2 to half the image size. The upward green arrows represent 3-D convolution with stride 2 to double the image size. The orange arrows represent copy operation. The results of green and orange operations are concatenated.

\subsection{Prediction and evaluation}

To apply the trained model to other datasets, they were first AC-PC-aligned by rigid-body registration to the MNI152 template, resampled to $0.7 \mathrm{~mm}$ isotropic resolution, and cropped to $64 \times 64 \times 64$ voxels centered at the average $\mathrm{Hb}$ location of the training data. Then, the trained model predicted the $\mathrm{Hb}$ segmentation. We then applied automated postprocessing on predicted segmentation to remove incorrect segmentation apart from $\mathrm{Hb}$ by removing disconnected regions from the segmentation, keeping two large regions.

The evaluation of the data with $\mathrm{T} 1 \mathrm{w} / \mathrm{T} 2 \mathrm{w}$ myelin content-based $\mathrm{Hb}$ segmentation (test data of HCP-YA S1200 and CamCAN data) included visual inspection, DSC, mean distance (MD, mean of surface distances from one segmentation's surface voxels to the other segmentation), and Hausdorff distance (HD), (Aspert et al., 2002), while only visual inspection was done for the Mount Sinai 7T datasets without myelin content-based $\mathrm{Hb}$ segmentation.

\subsection{MNI space transformation}

To compare average $\mathrm{Hb}$ segmentation between myelin content-based segmentation and deep learningbased segmentation, we transformed the segmentation of the test data to the MNI152 space using nonlinear transformations from the HCP pipeline.

\subsection{Data and code availability}

HCP-YA S1200 data are available at https://www.humanconnectome.org/study/hcp-young-adult. CamCAN data are available at https://www.cam-can.org/index.php?content=dataset.

Mount Sinai 7T data would remain confidential and would not be shared due to data ownership.

The codes for training, testing, and prediction, as well as HCP-YA S1200 subject lists of training, validation, and test sets, are available in a GitHub repository (https://github.com/joowon$\left.\mathrm{kim} / \mathrm{hb} \_s e g \_v n e t\right)$. The trained model used in this manuscript is available upon request due to its large size ( $>400 \mathrm{MB}$ ) to share via GitHub. 


\section{Results}

\subsection{Training of the $\mathrm{V}$-Net model}

The V-Net model training reached a steady state of loss function values ( 1 - DSC) less than 0.25 for both training and validation sets after about 30 epochs (Supplementary Fig 1). The training took about $30 \mathrm{~min}$ for each epoch, a total of 25 hours for 50 epochs. We chose the model with the lowest validation loss function value after the 30th epoch as our $\mathrm{Hb}$ segmentation model for the rest of the analysis (epoch 44, validation loss $=0.2048$ ).

\subsection{Test data and CamCAN data}

The predicted $\mathrm{Hb}$ segmentation from the test data $(n=157)$ and CamCAN data $(n=441)$ showed good agreement with the label $\mathrm{Hb}$ segmentation (Table 1 and Fig. 3 and 4). The DSCs were higher than 0.6 except for $3(1.9 \%)$ of the test data and 10 (2.3\%) of CamCAN data. The MDs were less than $0.5 \mathrm{~mm}$ for all of the test data and 20 (4.8\%) of CamCAN data, while the HDs were less than $3 \mathrm{~mm}$ except for 4 $(2.5 \%)$ of the test data and 24 (5.4\%) of CamCAN data. The threshold values of these measures were from our previous study about the reproducibility of myelin content-based $\mathrm{Hb}$ segmentation (Kim et al., 2018). The similarity of the test data was better than CamCAN data as expected because both the training data and the test data were HCP-YA S1200 data which were from the same scanner and scan parameters.

Table 1. Mean \pm standard deviation of label $\mathrm{Hb}$ volume and predicted $\mathrm{Hb}$ volume as well as their similarity comparison using dice similarity coefficient, mean distance, and Hausdorff distance from the test data and CamCAN data.

\section{Label volume $\left(\mathrm{mm}^{3}\right)$ \\ Prediction volume $\left(\mathrm{mm}^{3}\right)$ \\ Dice similarity coefficient \\ Mean distance $(\mathrm{mm})$ \\ Hausdorff distance $(\mathrm{mm})$}

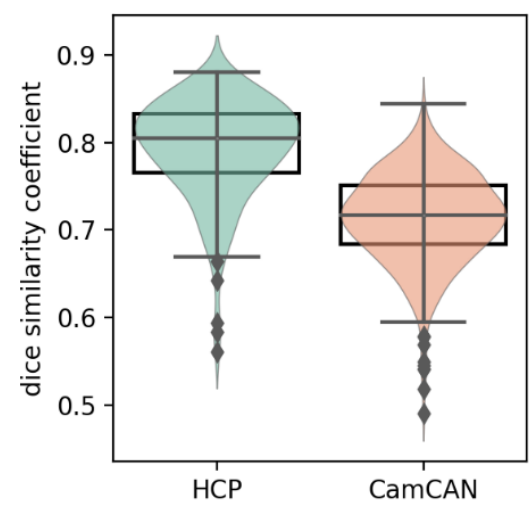

Test $(\mathbf{n}=157)$

$44.56 \pm 8.11$

$45.53 \pm 3.94$

$0.79 \pm 0.06$

$0.22 \pm 0.07$

$1.63 \pm 0.50$

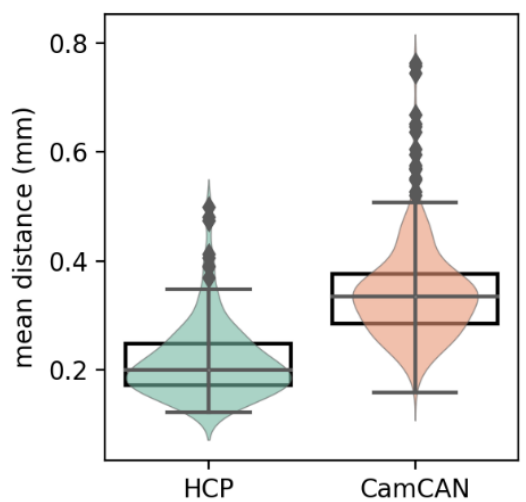

CamCAN ( $\mathbf{n}=\mathbf{4 4 1})$

$49.64 \pm 13.01$

$40.01 \pm 4.40$

$0.71 \pm 0.05$

$0.34 \pm 0.09$

$2.05 \pm 0.61$

Figure 3. Box plots on top of violin plots of dice similarity coefficient, mean distance, and Hausdorff distance from the test data $(n=157)$ and CamCAN data $(n=441)$.

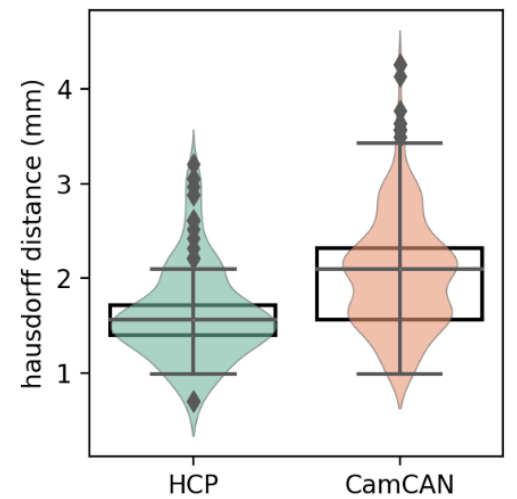



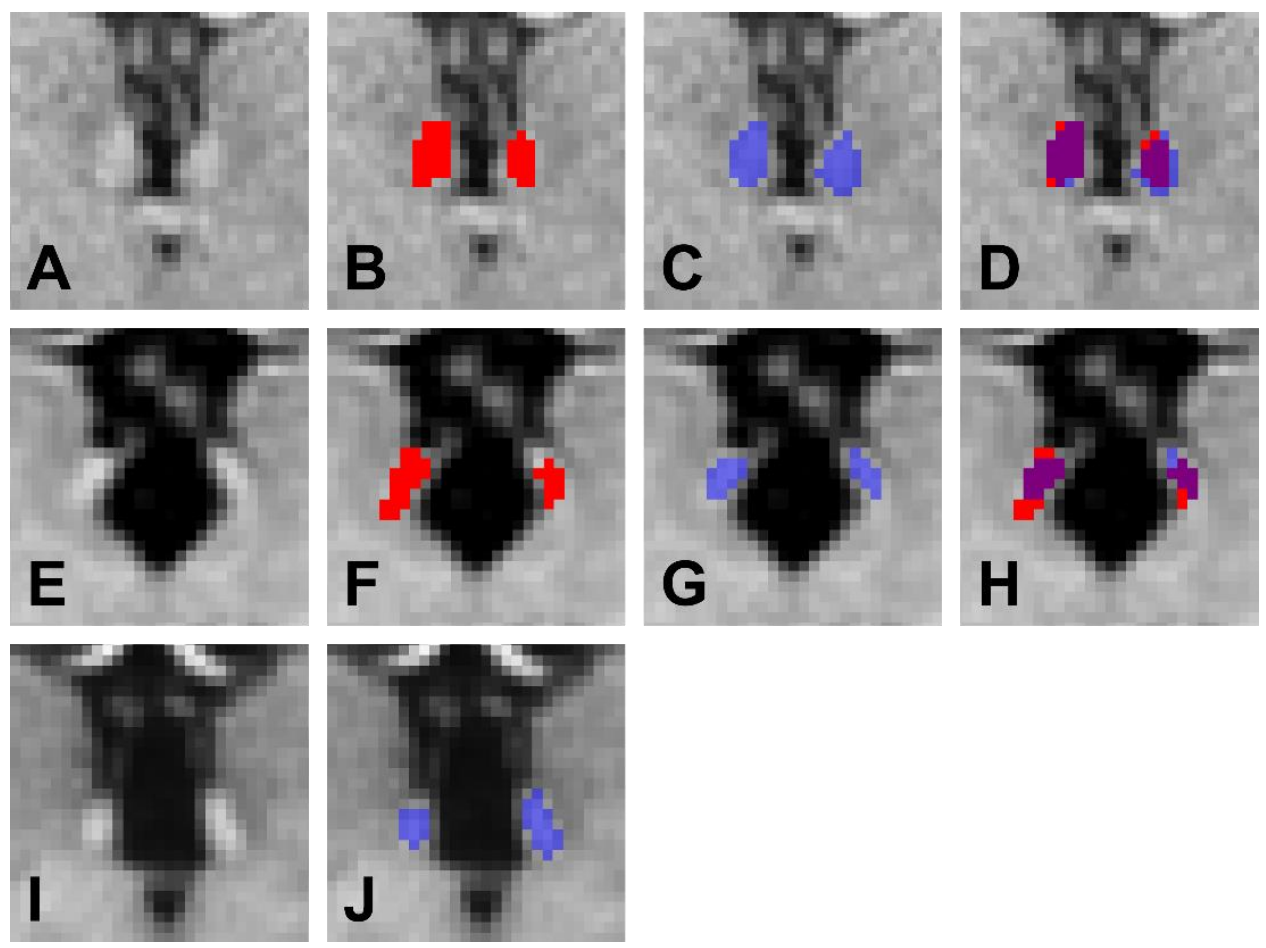

Figure 4. Representative coronal $\mathrm{T} 1 \mathrm{w}$ images zoomed around $\mathrm{Hb}(\mathrm{A}, \mathrm{E}, \mathrm{I}$, and $\mathrm{K})$ and $\mathrm{Hb}$ segmentation of label ( $B$ and $F$ ), prediction ( $C, G, J$, and $L$ ), and the comparison between label and prediction ( $D$ and $H$ ) from a test subject $(A-D, D S C=0.77, M D=0.24 \mathrm{~mm}, \mathrm{HD}=1.21 \mathrm{~mm})$, a CamCAN subject $(\mathrm{E}-\mathrm{H}, \mathrm{DSC}=0.74$, $\mathrm{MD}=0.29 \mathrm{~mm}, \mathrm{HD}=2.10 \mathrm{~mm})$, and a Mount Sinai $7 \mathrm{~T}$ subject (I-J). Red-colored regions are label $\mathrm{Hb}$ segmentation $(B, D, F$, and $H)$, blue regions are prediction $(C, D, G, H$, and $J)$, and purple regions are the intersection between label and prediction ( $D$ and $H$ ).

\subsection{Other data and previously failed cases}

The $\mathrm{Hb}$ was successfully segmented from Mount Sinai 7T data $(\mathrm{n}=5)$ (Fig. $4 \mathrm{I-J})$. For the subjects whose previous T1w/T2w myelin content-based segmentation failed due to insufficient contrast, the deep learning-based method successfully segmented the $\mathrm{Hb}$ (Supplementary Fig. 2).

\subsection{MNI space transformation}

The average $\mathrm{Hb}$ segmentation of the test data transformed to the MNI152 space is shown in Fig. X. Compared to the label segmentation (left, Fig. 5), the predicted segmentation (right, Fig. 5) was less over-estimated along the fasciculus retroflexus, a highly myelinated efferent fiber from the habenula (black arrow) and more overlapped across subjects. 




Figure 5. T1w MNI152 template images overlaid with average $\mathrm{Hb}$ label (left) and prediction (right) of the test data $(n=157)$ transformed to the MNI152 space. A black arrow points to the fasciculus retroflexus.

\section{Discussion}

We have demonstrated a deep learning-based $\mathrm{Hb}$ segmentation from T1 $\mathrm{w}$ images that was automated, objective, fast, robust, and reliable. A large amount of readily available labels were from our myelin content-based segmentation that used all T1w, T2w, and T1w/T2w ratio images in our previous studies. On the other hand, the training was on only T1w images, leading to prediction available on T1w images alone. Without requiring $\mathrm{T} 2 \mathrm{w}$ or $\mathrm{T} 1 \mathrm{w} / \mathrm{T} 2 \mathrm{w}$ ratio images, the deep learning-based segmentation showed comparable performance - high DSC and short MD/HD - to the myelin content-based segmentation. No manual work is necessary during deep learning-based segmentation prediction except for visual inspection.

Unlike other deep learning-based segmentation, our approach used different inputs when training (T1w images alone) from those when creating labels (T1w, T2w, and T1w/T2w images). Due to this difference, we cannot expect identical or near-identical predictions to labels even in training sets. Nevertheless, our DSC, MD, and HD are comparable to other deep learning-based segmentation methods for spinal cord gray matter (Perone et al., 2018) or hippocampus (Carmo et al., 2021), which is more than expected.

We highly recommend a visual inspection of each subject's segmentation to find obvious erroneous segmentation, although there were no such cases in our data. The postprocessing to remove off-Hbregion was necessary because the predicted $\mathrm{Hb}$ regions sometimes (less than $1 \%$ ) contain voxels far off the $\mathrm{Hb}$ region. After the postprocessing, visual inspection became straightforward to check 5 to 10 slices around the $\mathrm{Hb}$ region.

In addition to the high similarity in the test data, this new approach also showed robustness. It successfully segmented the $\mathrm{Hb}$ of the subjects that myelin content-based segmentation failed to segment $\mathrm{Hb}$. We expect that this is not uncommon because myelin content-based segmentation requires high-quality, well-aligned T1w and T2w images; if either image quality is compromised by subject motion or the alignment is imperfect, the T1w/T2w image quality decreases, leading to unsatisfactory segmentation using the myelin content-based method.

When segmenting other datasets, we resampled input images to the same resolution as the training data. This resampling ensures similar sizes of inputs but introduces blurry effects. Using native 
resolution might be viable if we train various resolution data, but it is not straightforward to tell which way is preferred in terms of segmentation performance.

The training data were of young adults scanned with the same scanner and imaging parameters, leading to high performance on similar data types but lower performance on others. Adding another training data with a wide age range, multiple scanners, and different imaging parameters may increase the robustness of the segmentation.

The training labels were based on myelin content that is heterogeneous in Hb (Díaz et al., 2011). Therefore, the myelin content-based segmentation sometimes underestimates $\mathrm{Hb}$ near a less myelinated area but overestimates along highly myelinated fibers connected to the $\mathrm{Hb}$ (e.g., fasciculus retroflexus or stria medullaris). We did not observe group-wise overestimation along fasciculus retroflexus in the deep learning-based segmentation. This is likely due to the lack of T2w or T1w/T2w images, which accentuates the myelin contrast. This could indicate that the deep learning-based segmentation relies less on intensities but more on shapes than myelin content-based segmentation.

\section{Acknowledgment}

We are grateful to Dr. Sophia Frangou (currently at University of British Columbia) at Icahn School of Medicine at Mount Sinai for sharing the Mount Sinai 7T data.

Data for deep learning were provided by the Human Connectome Project, WU-Minn Consortium (Principal Investigators: David Van Essen and Kamil Ugurbil; 1U54MH091657) funded by the $16 \mathrm{NIH}$ Institutes and Centers that support the NIH Blueprint for Neuroscience Research; and by the McDonnell Center for Systems Neuroscience at Washington University.

Data collection and sharing for $1 \mathrm{~mm}$ isotropic resolution data was provided by the Cambridge Centre for Ageing and Neuroscience (CamCAN). CamCAN funding was provided by the UK Biotechnology and Biological Sciences Research Council (grant number BB/H008217/1), together with support from the UK Medical Research Council and University of Cambridge, UK. 


\section{References}

Aspert, N., Santa-Cruz, D., Ebrahimi, T., 2002. MESH: measuring errors between surfaces using the Hausdorff distance, in: 2002 IEEE International Conference on Multimedia and Expo, 2002. ICME '02. Proceedings. Presented at the 2002 IEEE International Conference on Multimedia and Expo, 2002. ICME '02. Proceedings, pp. 705-708 vol.1. https://doi.org/10.1109/ICME.2002.1035879

Benarroch, E.E., 2015. Habenula Recently recognized functions and potential clinical relevance. Neurology 85, 992-1000. https://doi.org/10.1212/WNL.0000000000001937

Carceller-Sindreu, M., de Diego-Adeliño, J., Serra-Blasco, M., Vives-Gilabert, Y., Martí;n-Blanco, A., Puigdemont, D., Álvarez, E., Pérez, V., Portella, M.J., 2015. Volumetric MRI study of the habenula in first episode, recurrent and chronic major depression. European Neuropsychopharmacology 25, 2015-2021. https://doi.org/10.1016/j.euroneuro.2015.08.009

Carmo, D., Silva, B., Yasuda, C., Rittner, L., Lotufo, R., 2021. Hippocampus segmentation on epilepsy and Alzheimer's disease studies with multiple convolutional neural networks. Heliyon 7, e06226. https://doi.org/10.1016/j.heliyon.2021.e06226

Chollet, F., others, 2015. Keras.

Díaz, E., Bravo, D., Rojas, X., Concha, M.L., 2011. Morphologic and immunohistochemical organization of the human habenular complex. J. Comp. Neurol. 519, 3727-3747. https://doi.org/10.1002/cne.22687

Dice, L.R., 1945. Measures of the Amount of Ecologic Association Between Species. Ecology 26, 297-302. https://doi.org/10.2307/1932409

Ely, B.A., Stern, E.R., Kim, J., Gabbay, V., Xu, J., 2019. Detailed mapping of human habenula resting-state functional connectivity. Neurolmage 200, 621-634. https://doi.org/10.1016/j.neuroimage.2019.06.015

Ely, B.A., Xu, J., Goodman, W.K., Lapidus, K.A., Gabbay, V., Stern, E.R., 2016. Resting-state functional connectivity of the human habenula in healthy individuals: Associations with subclinical depression. Hum Brain Mapp 37, 2369-2384. https://doi.org/10.1002/hbm.23179

Fore, S., Palumbo, F., Pelgrims, R., Yaksi, E., 2018. Information processing in the vertebrate habenula. Seminars in Cell \& Developmental Biology, The cancer secretome and secreted biomarkers 78, 130-139. https://doi.org/10.1016/j.semcdb.2017.08.019

Germann, J., Gouveia, F.V., Martinez, R.C.R., Zanetti, M.V., de Souza Duran, F.L., Chaim-Avancini, T.M., Serpa, M.H., Chakravarty, M.M., Devenyi, G.A., 2020. Fully Automated Habenula Segmentation Provides Robust and Reliable Volume Estimation Across Large Magnetic Resonance Imaging Datasets, Suggesting Intriguing Developmental Trajectories in Psychiatric Disease. Biological Psychiatry: Cognitive Neuroscience and Neuroimaging 5, 923-929.

https://doi.org/10.1016/j.bpsc.2020.01.004

Glasser, M.F., Sotiropoulos, S.N., Wilson, J.A., Coalson, T.S., Fischl, B., Andersson, J.L., Xu, J., Jbabdi, S., Webster, M., Polimeni, J.R., Van Essen, D.C., Jenkinson, M., WU-Minn HCP Consortium, 2013. The minimal preprocessing pipelines for the Human Connectome Project. Neuroimage 80, 105124. https://doi.org/10.1016/j.neuroimage.2013.04.127

He, K., Zhang, X., Ren, S., Sun, J., 2015. Delving Deep into Rectifiers: Surpassing Human-Level Performance on ImageNet Classification. arXiv:1502.01852 [cs].

Hikosaka, O., 2010. The habenula: from stress evasion to value-based decision-making. Nat Rev Neurosci 11, 503-513. https://doi.org/10.1038/nrn2866

Jha, M.K., Kim, J., Kenny, P.J., Chin Fatt, C., Minhajuddin, A., Salas, R., Ely, B.A., Klein, M., Abdallah, C.G., Xu, J., Trivedi, M.H., 2021. Smoking status links habenular volume to glycated hemoglobin: Findings from the Human Connectome Project-Young Adult. Psychoneuroendocrinology 131, 105321. https://doi.org/10.1016/j.psyneuen.2021.105321 
Kim, J., Xu, J., 2018. Trajectory of Human Habenula Volume and Location Through Adult Lifespan., in: The International Society for Magnetic Resonance in Medicine (ISMRM) Workshop on Advanced Neuro MR: Best Practices for Technical Implementation. Presented at the The International Society for Magnetic Resonance in Medicine (ISMRM) Workshop on Advanced Neuro MR: Best Practices for Technical Implementation., Seoul, South Korea.

Kim, J.-W., Naidich, T.P., Ely, B.A., Yacoub, E., De Martino, F., Fowkes, M.E., Goodman, W.K., Xu, J., 2016. Human habenula segmentation using myelin content. Neurolmage 130, 145-156. https://doi.org/10.1016/j.neuroimage.2016.01.048

Kim, J.-W., Naidich, T.P., Joseph, J., Nair, D., Glasser, M.F., O’halloran, R., Doucet, G.E., Lee, W.H., Krinsky, H., Paulino, A., Glahn, D.C., Anticevic, A., Frangou, S., Xu, J., 2018. Reproducibility of myelin content-based human habenula segmentation at 3 Tesla. Hum Brain Mapp 39, 30583071. https://doi.org/10.1002/hbm.24060

Kingma, D.P., Ba, J., 2017. Adam: A Method for Stochastic Optimization. arXiv:1412.6980 [cs].

Lawson, R.P., Drevets, W.C., Roiser, J.P., 2013. Defining the habenula in human neuroimaging studies. Neuroimage 64, 722-727. https://doi.org/10.1016/j.neuroimage.2012.08.076

Lim, S.-H., Yoon, J., Kim, Y.J., Kang, C.-K., Cho, S.-E., Kim, K.G., Kang, S.-G., 2021. Reproducibility of automated habenula segmentation via deep learning in major depressive disorder and normal controls with 7 Tesla MRI. Sci Rep 11, 13445. https://doi.org/10.1038/s41598-021-92952-z

Marques, J.P., Kober, T., Krueger, G., van der Zwaag, W., Van de Moortele, P.-F., Gruetter, R., 2010. MP2RAGE, a self bias-field corrected sequence for improved segmentation and T1-mapping at high field. Neurolmage 49, 1271-1281. https://doi.org/10.1016/j.neuroimage.2009.10.002

Martín Abadi, Ashish Agarwal, Paul Barham, Eugene Brevdo, Zhifeng Chen, Craig Citro, Greg S. Corrado, Andy Davis, Jeffrey Dean, Matthieu Devin, Sanjay Ghemawat, lan Goodfellow, Andrew Harp, Geoffrey Irving, Michael Isard, Jia, Y., Rafal Jozefowicz, Lukasz Kaiser, Manjunath Kudlur, Josh Levenberg, Dandelion Mané, Rajat Monga, Sherry Moore, Derek Murray, Chris Olah, Mike Schuster, Jonathon Shlens, Benoit Steiner, Ilya Sutskever, Kunal Talwar, Paul Tucker, Vincent Vanhoucke, Vijay Vasudevan, Fernanda Viégas, Oriol Vinyals, Pete Warden, Martin Wattenberg, Martin Wicke, Yuan Yu, Xiaoqiang Zheng, 2015. TensorFlow: Large-Scale Machine Learning on Heterogeneous Systems.

Milletari, F., Navab, N., Ahmadi, S.-A., 2016. V-Net: Fully Convolutional Neural Networks for Volumetric Medical Image Segmentation, in: 2016 Fourth International Conference on 3D Vision (3DV). Presented at the 2016 Fourth International Conference on 3D Vision (3DV), pp. 565-571. https://doi.org/10.1109/3DV.2016.79

Namboodiri, V.M.K., Rodriguez-Romaguera, J., Stuber, G.D., 2016. The habenula. Current Biology 26, R873-R877. https://doi.org/10.1016/j.cub.2016.08.051

NVIDIA, 2019. CUDA 10.1.

Perone, C.S., Calabrese, E., Cohen-Adad, J., 2018. Spinal cord gray matter segmentation using deep dilated convolutions. Sci Rep 8, 5966. https://doi.org/10.1038/s41598-018-24304-3

Ranft, K., Dobrowolny, H., Krell, D., Bielau, H., Bogerts, B., Bernstein, H.-G., 2010. Evidence for structural abnormalities of the human habenular complex in affective disorders but not in schizophrenia. Psychol Med 40, 557-567. https://doi.org/10.1017/S0033291709990821

Ronneberger, O., Fischer, P., Brox, T., 2015. U-Net: Convolutional Networks for Biomedical Image Segmentation, in: Navab, N., Hornegger, J., Wells, W.M., Frangi, A.F. (Eds.), Medical Image Computing and Computer-Assisted Intervention - MICCAI 2015, Lecture Notes in Computer Science. Springer International Publishing, Cham, pp. 234-241. https://doi.org/10.1007/978-3319-24574-4_28

Savitz, J.B., Nugent, A.C., Bogers, W., Roiser, J.P., Bain, E.E., Neumeister, A., Zarate, C.A., Manji, H.K., Cannon, D.M., Marrett, S., Henn, F., Charney, D.S., Drevets, W.C., 2011. Habenula volume in 
bipolar disorder and major depressive disorder: a high-resolution magnetic resonance imaging study. Biol. Psychiatry 69, 336-343. https://doi.org/10.1016/j.biopsych.2010.09.027

Schafer, M., Kim, J.-W., Joseph, J., Xu, J., Frangou, S., Doucet, G.E., 2018. Imaging Habenula Volume in Schizophrenia and Bipolar Disorder. Front. Psychiatry 9. https://doi.org/10.3389/fpsyt.2018.00456

Schmidt, F.M., Schindler, S., Adamidis, M., Strauß, M., Tränkner, A., Trampel, R., Walter, M., Hegerl, U., Turner, R., Geyer, S., Schönknecht, P., 2016. Habenula volume increases with disease severity in unmedicated major depressive disorder as revealed by 7T MRI. Eur Arch Psychiatry Clin Neurosci 1-9. https://doi.org/10.1007/s00406-016-0675-8

Shafto, M.A., Tyler, L.K., Dixon, M., Taylor, J.R., Rowe, J.B., Cusack, R., Calder, A.J., Marslen-Wilson, W.D., Duncan, J., Dalgleish, T., Henson, R.N., Brayne, C., Matthews, F.E., 2014. The Cambridge Centre for Ageing and Neuroscience (Cam-CAN) study protocol: a cross-sectional, lifespan, multidisciplinary examination of healthy cognitive ageing. BMC Neurol 14. https://doi.org/10.1186/s12883-014-0204-1

Taylor, J.R., Williams, N., Cusack, R., Auer, T., Shafto, M.A., Dixon, M., Tyler, L.K., Cam-CAN, Henson, R.N., 2017. The Cambridge Centre for Ageing and Neuroscience (Cam-CAN) data repository: Structural and functional MRI, MEG, and cognitive data from a cross-sectional adult lifespan sample. Neurolmage, Data Sharing Part II 144, 262-269. https://doi.org/10.1016/j.neuroimage.2015.09.018

Van Essen, D.C., Ugurbil, K., Auerbach, E., Barch, D., Behrens, T.E.J., Bucholz, R., Chang, A., Chen, L., Corbetta, M., Curtiss, S.W., Della Penna, S., Feinberg, D., Glasser, M.F., Harel, N., Heath, A.C., Larson-Prior, L., Marcus, D., Michalareas, G., Moeller, S., Oostenveld, R., Petersen, S.E., Prior, F., Schlaggar, B.L., Smith, S.M., Snyder, A.Z., Xu, J., Yacoub, E., 2012. The Human Connectome Project: A data acquisition perspective. Neurolmage, Connectivity 62, 2222-2231. https://doi.org/10.1016/j.neuroimage.2012.02.018 